\title{
Fatal Acute Pulmonary Aspergillosis in Two Children of One Family
}

\author{
M. K. STRELLING, K. RHANEY, D. A. R. SIMMONS, and J. THOMSON \\ From the Royal Infirmary, Dundee, and the Departments of Child Health and of Bacteriology, \\ Queen's College, University of St. Andrews
}

Invasive pulmonary aspergillosis is uncommon in man and is rare as a cause of death in children. When diffuse pulmonary infection with aspergillus has developed it has nearly always complicated a pre-existing disorder, either of the lungs themselves or one of a more generalized nature (Symmers, 1964). We present, therefore, an account of fatal pulmonary aspergillosis affecting a child and her infant sister, who had both previously been in normal health. The bacteriological investigation of the infected tissues and of the children's environment is also described.

\section{Case Reports}

Case 1. A girl of 4 years was admitted to hospital on October 18, 1962, with a two-day history of breathlessness, fever, and slight cough. She had previously been well and had not suffered from any of the common infectious diseases of childhood, though a paronychia had been pricked and squeezed by her father three weeks beforehand.

On admission she was pale, ill, and dyspnoeic with a temperature of $100^{\circ} \mathrm{F} .\left(37 \cdot 8^{\circ} \mathrm{C}\right.$. $)$. Examination of the chest revealed a respiratory rate of $60 /$ minute, a resonant percussion note and moderately reduced breath sounds. No adventitious sounds were heard then or subsequently and no other abnormal physical signs were present. Chest radiographs showed a patchy infiltration of the lung fields which was most conspicuous centrally, but did not involve the apices. In subsequent radiographs the opacities had spread to involve the peripheral zones as well (Fig. 1). Blood examination revealed a total white count of $30,000 /$ c.mm. with a neutrophil leucocytosis and a moderate degree of hypochromic anaemia with a haemoglobin of $11 \cdot 2 \mathrm{~g} . / 100 \mathrm{ml}$. A Mantoux tuberculin test of $1: 1,000$ was negative. Repeated attempts to identify the cause of the pulmonary disease were unsuccessful. The flora of the throat and stool yielded no recognizable pathogen, and cultures of blood, urine, and cerebrospinal fluid were sterile. A needle biopsy of the lung was performed on the last day of her illness, but no organisms were demonstrated by microscopy, and cultures of the aspirated material were sterile.

Received April 30, 1965.
Initial treatment was with penicillin $(500,000$ units i.m. 12-hourly) and sulphadimidine (5 g. over 6 days); the next day, methicillin (15 g. i.m. over 5 days) was substituted for penicillin, but no response was observed, so erythromycin ( 6 g. over six days), cloxacillin ( 3 g. over 3 days), and on the last day, a combination of tetracycline and nystatin ('Mysteclin', Squibb and Co.) were used empirically.

The child's clinical condition did not improve and her respiration remained rapid, fluctuating between 60 and $100 /$ minute. Fever, however, was slight, the highest temperature recorded being $100^{\circ} \mathrm{F}$. $\left(37 \cdot 8^{\circ} \mathrm{C}\right.$.). She finally collapsed and died with a bilateral spontaneous pneumothorax on the 14th day of her illness.

NeCROPSY. External examination: the body was that of a slender female child. No sign of the paronychia remained. The superficial lymph nodes were not enlarged. There was no jaundice or oedema.

Internal examination: disease was confined to the thoracic organs. A tension pneumothorax was present on the right and a partial pneumothorax on the left. The upper respiratory passages were not inflamed and contained a little serous fluid. The lungs (right $291 \mathrm{~g}$., left $183 \mathrm{~g}$.) were heavy and showed widespread induration or consolidation (Fig. 2) of a rubbery texture. Their pleural surfaces were congested and mottled and there were a few petechiae. On section the parenchyma showed widespread consolidation which was diffuse in the posterior parts and nodular anteriorly, the nodules being of pinkish colour and measuring $1-3 \mathrm{~cm}$. in diameter. Some lobules were emphysematous, and there was interstitial emphysema which had extended to the pleura and to the mediastinum. The bronchopulmonary and tracheo-bronchial lymph nodes were considerably enlarged but were discrete and showed no specific changes. The heart showed slight dilatation of the right atrium and ventricle. The abdominal organs were healthy: the mesenteric lymph nodes were yellowishbrown. The central nervous system was not examined.

Histology. Lungs: There was widespread inflammation of the air spaces and of the intralobular bronchial system and interstitial tissue so that the architecture of the lung was somewhat obscured (Fig. 3). The inter- 


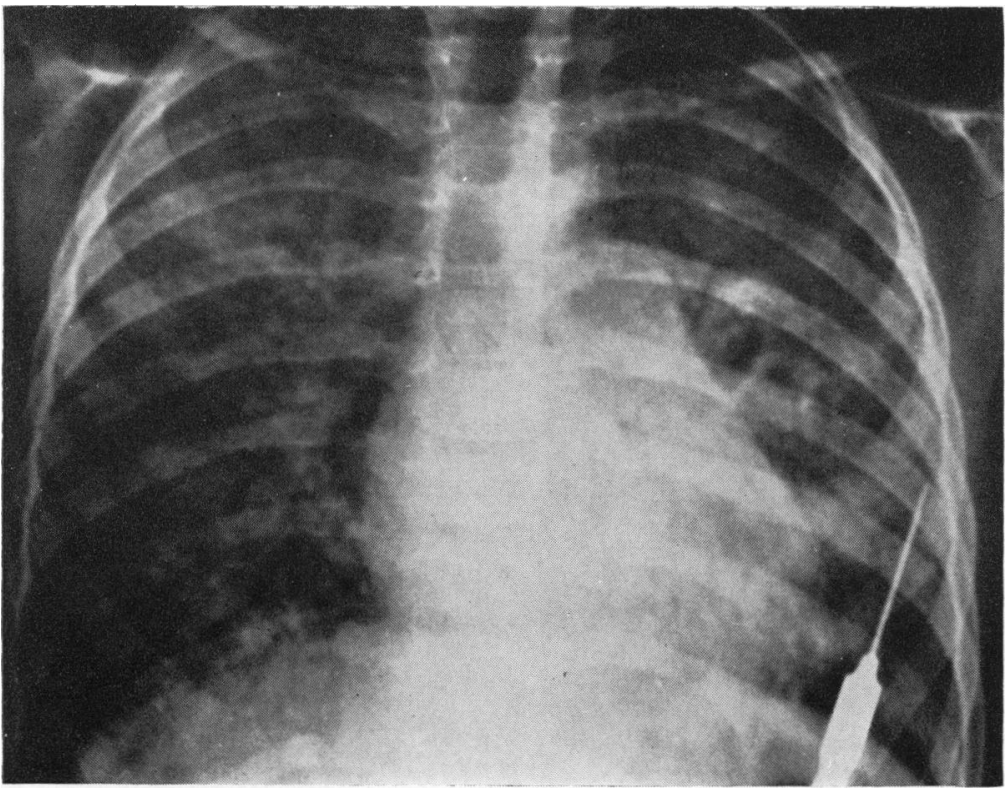

FIG. 1.-Radiograph of Case 1 showing widespread, small, fluffy-edged opacities.

stitial inflammation was intense and produced great thickening of the framework, the infiltrating cells being predominantly plasma cells with some lymphocytes and a few polymorphs. The air spaces were reduced in size

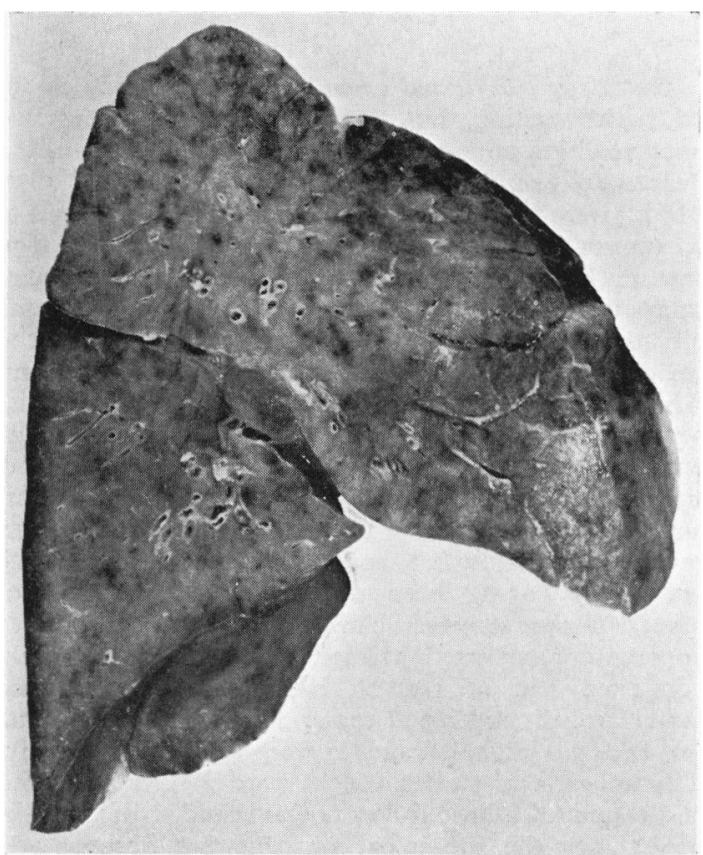

Fig. 2.-Case 1. Lung (after fixation) showing diffuse consolidation. and had a prominent epithelial lining. Most of the air spaces and terminal respiratory passages contained distinctive granulomatous lesions of varied shape and size. Some of the lesions consisted solely of histiocytic cells with a few multi-nucleated giant cells (thus resembling non-caseous tubercle follicles) but many had a central core of polymorphs forming micro-abscesses. Most of the polymorphs were neutrophils, a few were eosinophils, and in many lesions they were pyknotic. Some of these granulomata might also have been interstitial. They contained fragments of fungus (see Case 2), but these were scanty and were stained most clearly by a modification of Gomori's methenaminesilver method (Grocott, 1955)-using haemalumtartrazine as a counterstain - and less easily by the PAS stain or by various modifications of Gram's method. Elsewhere some of the air spaces contained foamy histiocytes and others contained polymorphs or were haemorrhagic; some were emphysematous.

The broncho-pulmonary lymph nodes and the spleen showed a non-specific chronic inflammatory reaction; they contained scanty fungi.

The liver contained occasional small foci of necrosis and histiocytic infiltration, but fungi were scanty and were apparently unrelated to the focal lesions.

Clusters of histiocytes in the mesenteric lymph nodes, in the spleen, and in the wall of the small intestine and in the liver were swollen with a yellowish intracytoplasmic pigment which stained for lipofuscin.

Other organs and tissues showing no specific lesions were: trachea, submandibular salivary gland, thyroid, thymus, heart, oesophagus, pancreas, adrenal gland, kidney, ovary, uterus, stomach, skin and muscle of abdominal wall, spinal cord, and bone-marrow. 


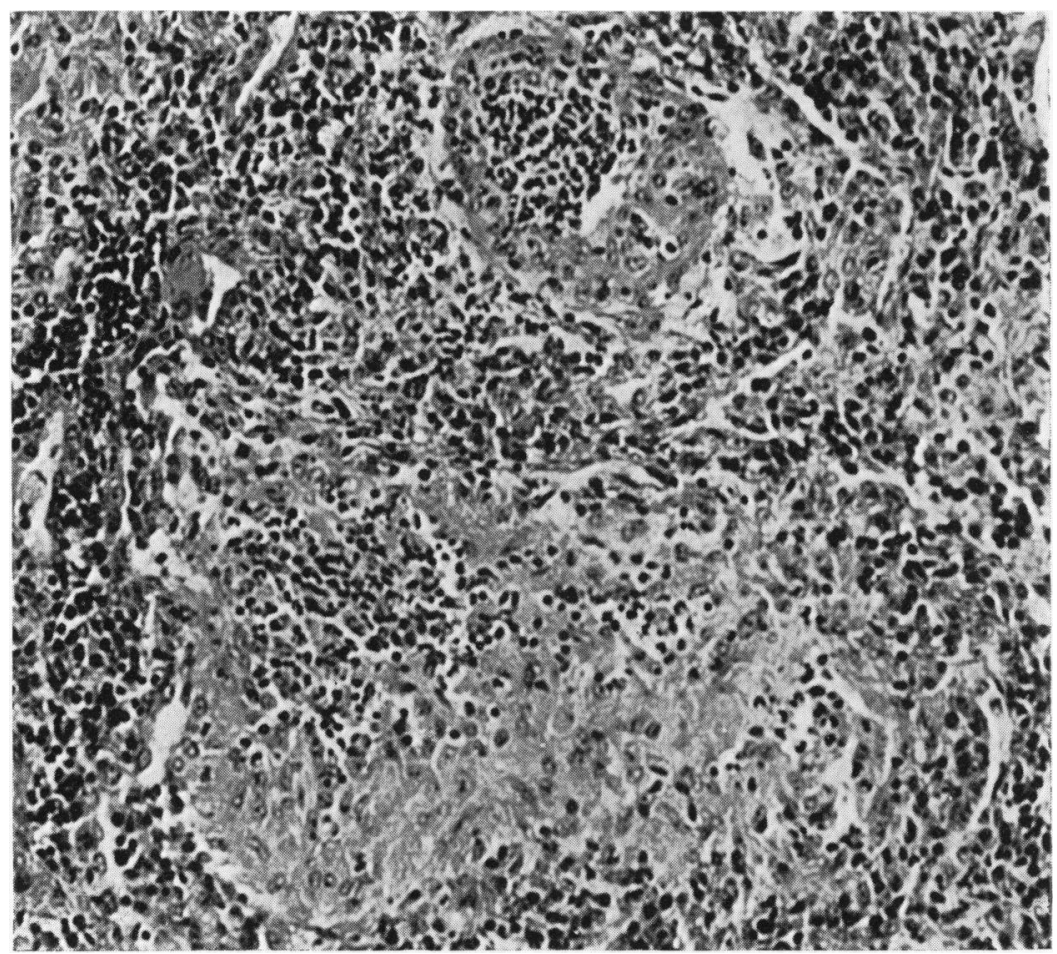

FIg. 3.-Lung of Case 1 showing the intense interstitial inflammation and the granulomatous lesions. (Haematoxylin and eosin. $\times$ 300.)

Case 2. A few days before the first patient died, her infant sister, aged 14 months, also became ill with fever and breathlessness and was admitted to hospital. Like her sister she had had a paronychia drained by her father three weeks beforehand but apart from this had previously been well. On admission her temperature was also only $100^{\circ} \mathrm{F}$. $\left(37 \cdot 8^{\circ}\right.$ C. $)$. Her respirations were rapid and grunting with intercostal recession, and though physical signs were confined to diminished breath sounds, radiographs revealed widespread fluffy-edged pulmonary opacities which were somewhat finer than those of her sister; these became increasingly diffuse on subsequent films. There was a neutrophil leucocytosis of 24,000/ c.mm., a haemoglobin of $9.9 \mathrm{~g} . / 100 \mathrm{ml}$., and a negative Heaf tuberculin test. Her $\gamma$-globulin level was 1,280 mg. $/ 100 \mathrm{ml}$. No recognized pathogens were grown from swabs of nose, throat, or rectum, and Sabouraud's medium yielded only a few colonies of monilia from a throat swab taken on the ninth day after admission. The illness in this child also pursued an inexorable course despite the administration of tetracycline ( $4 \mathrm{~g}$. over 7 days), chloramphenicol ( 2 g. over 2 days), and streptomycin (800 mg.) and isoniazid (200 mg.) both over 2 days. Prednisone (total dose of $270 \mathrm{mg}$.) was also given for 8 days. During the last 48 hours congestive heart failure developed, but despite digitalization she, too, died on the 14th day of her illness.
NeCropsy. External examination: The body showed slight wasting, but there were no other external abnormalities, no paronychia, and no lymphadenopathy.

Internal exarination: The lungs (right $243 \mathrm{~g}$., left $218 \mathrm{~g}$.) were massively infiltrated with nodular lesions of an unusual kind and were extremely heavy and of firm texture (Fig. 4). The pleural surfaces were mottled and uneven, the focal lesions and regions of emphysema being elevated and pale, so that they were in striking contrast to the congested, reddish purple lung tissue between them. The firm, dry, white nodular lesions were widely disseminated throughout all lobes and were almost confluent in some regions. They were of varied size and shape, the smallest being approximately $0.1 \mathrm{~cm}$. in diameter, and resembled miliary tubercles, while the largest measured between 0.5 and $1 \mathrm{~cm}$. in diameter, these apparently having been produced by confluence of several smaller lesions. The relatively small amount of apparently unaffected pulmonary tissue was intensely congested and of reddish brown appearance. The larger bronchi and blood vessels appeared to be normal but their peripheral branches were difficult to identify. The upper respiratory passages were slightly congested and contained a little frothy mucoid fluid. In the right upper lobe the sub-apical segment was fibrosed and contracted so that a deep fissure had developed on its lateral surface and from this fissure a thick white fibrous 


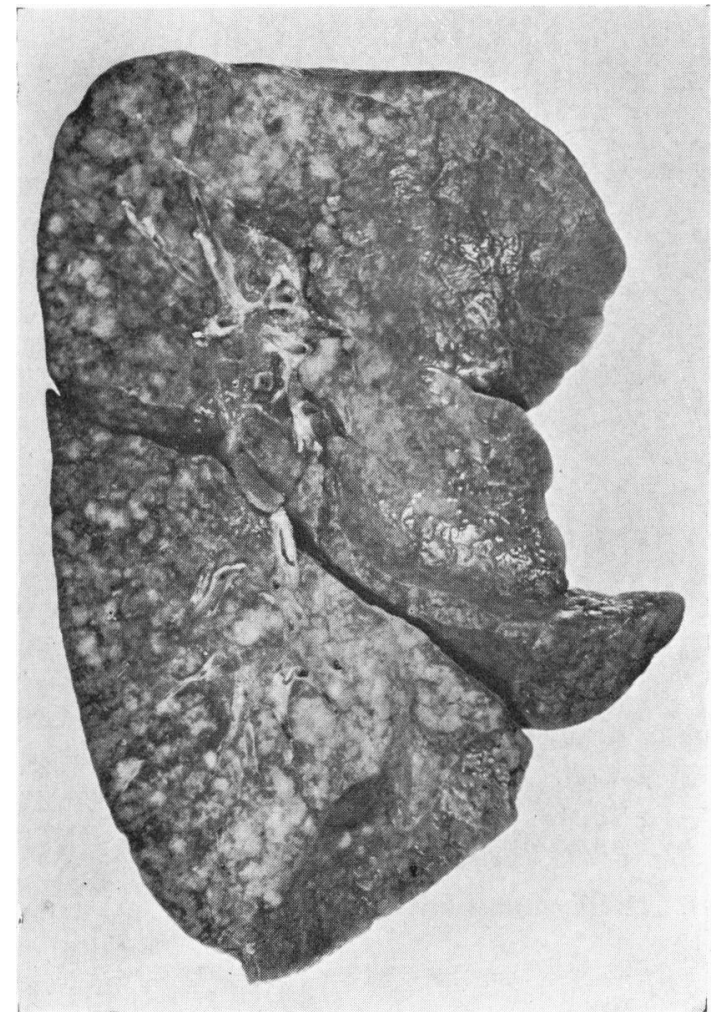

FIG. 4.-Lung of Case 2 showing widespread focal infiltration.

adhesion extended to the postero-lateral wall of the thorax. The scarred lung showed no specific feature on section, and there were no changes in the related lymph nodes to suggest a previous episode of primary tuberculosis.

The mediastinal lymph nodes: the broncho-pulmonary and the inferior and superior tracheo-bronchial lymph nodes were considerably enlarged, measuring up to 2.5 $\mathrm{cm}$. in diameter. They were discrete and fleshy and of reddish brown colour. In one of the broncho-pulmonary nodes related to the right lung there was a small white focus which appeared to be a metastatic fungal lesion, but no specific changes could be detected in any of the other nodes. The lymphadenopathy also involved the para-tracheal and para-oesophageal nodes.

The heart $(60 \mathrm{~g}$.) was slightly enlarged and there appeared to be slight dilatation and hypertrophy of the right ventricle. The spleen $(24.5 \mathrm{~g}$.) was of average size and normal appearance.

The alimentary, genito-urinary, and central nervous system appeared to be normal. The bone-marrow in the ribs and vertebral bodies was of normal appearance.

HISTOLOGY. The lungs showed a more florid inflammatory picture than that found in the older sister, and fungi were so abundant that they could be demonstrated quite easily in routine $H$. and $E$. sections as well as by specific methods (Fig. 5 and 6).

The inflammation was virtually diffuse throughout the lobules involving the intralobular bronchial system as well as the air spaces. It was associated with widespread inflammatory infiltration of the interstitial tissue as well as with cellular exudation into the air spaces and bronchial tree.

The exudate consisted of polymorphs and granulomata, the latter consisting of a thin peripheral shell of eosinophilic epithelioid cells with a large central core of polymorphs. Some of the epithelioid cells had become transformed into multinucleated giant cells.

The interstitial reaction, which had expanded and loosened the framework of the lung, consisted of histiocytic proliferation and, in places, of infiltration with lymphocytes and plasma cells. Local cellular infiltration was also present in the septa and pleura. The inflammation had given rise to the production of new reticulin fibres, notably in the granulomatous lesions, and there were also small foci of fibrosis. Focal devitalization of inflammatory tissue occurred in places, sometimes following thrombosis of alveolar capillaries, and haemorrhage had developed in several regions. Even the noninflamed portions of the lobules were congested and very oedematous, as were the interlobular septa and pleura.

Fungi were abundant in comparison with Case 1 and, as stated earlier, they were readily seen in routine $\mathrm{H}$. and $\mathrm{E}$. sections as well as being clearly demonstrated by Grocott's modification of the methenamine-silver and by the PAS and Gram methods.

Mediastinal lymph nodes showed a non-specific lymphadenitis and contained fungi. One bronchopulmonary node contained micro-abscesses with a surrounding shell of epithelioid cells similar to those seen in the lung.

The spleen showed a non-specific response to infection and contained numerous fungi. The kidney showed small lesions of nephrocalcinosis near the apex of the pyramids. The bone-marrow showed normal active haemopoiesis.

No abnormality was present in the liver, pancreas, adrenal glands, small intestine, and central nervous system.

\section{Environment}

The almost simultaneous occurrence of a closely similar illness in the sisters suggested a common source of infection from their farm environment. During the preceding months these children had played for long periods in a barn in which were stored both natural and artificial manure, linseed oil cattle cake, and grain. From such materials aspergillus can be recovered (Thom and Raper, 1945). As reported in the bacteriological section, a growth of Aspergillus fumigatus was in fact obtained from the cattle cake, and the strain of this fungus proved to be indistinguishable from those that were subsequently isolated at necropsy from both children.

\section{Family History}

Their parents, who are farmers, and an elder son aged 6 years, have remained well both clinically and radiologic- 


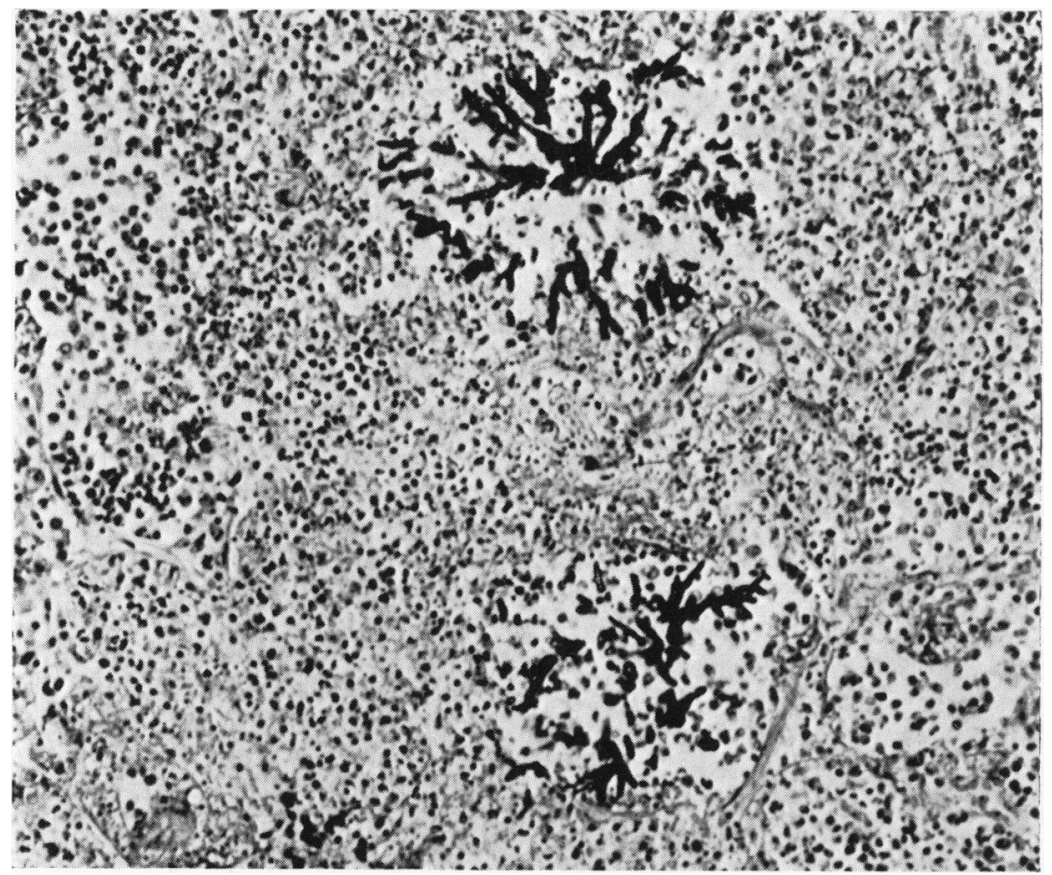

FIG. 5.-Lung of Case 2 showing the fungi. (Methenamine-silver. $\quad \times 230$.

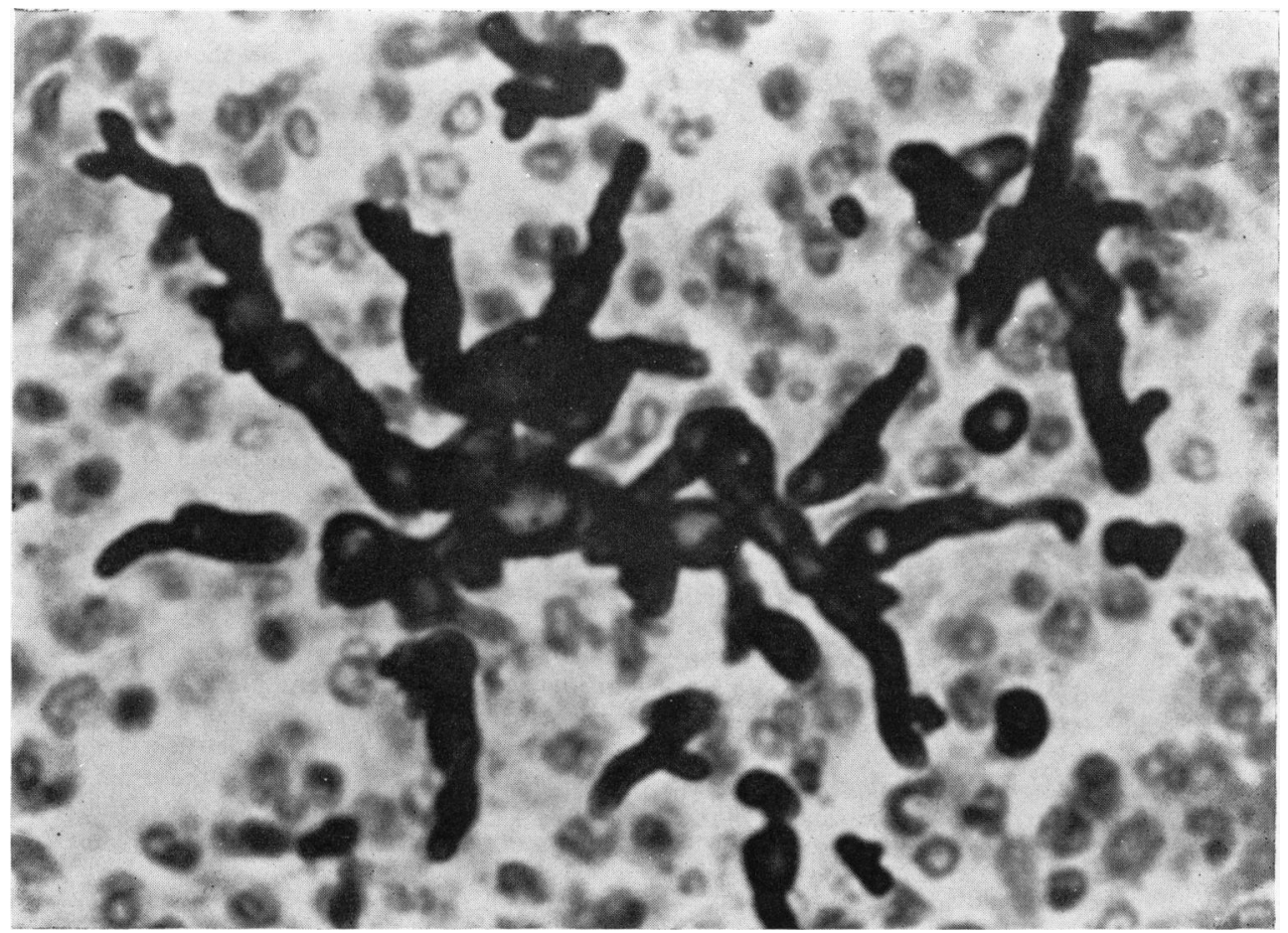

Fig. 6.-Lung of Case 2 showing the fungi. (Methenamine-silver. $\quad \times 1045$. 
ally. Electrophoresis of the plasma proteins did not show a deficiency of $\gamma$-globulin in any member of the family.

\section{Bacteriology}

Case 1. The flora of the throat and gut were normal. All cultures of urine, cerebrospinal fluid, and blood remained sterile. In a small piece of tissue obtained by attempted needle biopsy of the lung, no inflammatory cells or organisms, including acid-alcohol fast bacilli, were demonstrated, and all cultures, including those for Mycobacterium tuberculosis and $A$. fumigatus, remained sterile. No growth was obtained aerobically or anaerobically in bronchial and lung swabs received post mortem, and no fungi were isolated. Impression smears of postmortem lung showed no inflammatory cells or organisms, including acid-alcohol-fast bacilli. Routine media, including Sabouraud's, remained sterile, but $A$. fumigatus was isolated from a Lowenstein-Jensen slope after 10 days incubation at $37^{\circ} \mathrm{C}$.

Case 2. The flora of the throat and nose were normal. All cultures of urine and blood remained sterile. No intestinal pathogens, bacterial or viral, were isolated; no cytopathogenic effect was produced by stool inoculum either with monkey kidney or with primary human amnion cultures. The serum produced no demonstrable agglutination of standard Salmonella typhi, S. paratyphi B, or Brucella abortus suspensions. A few colonies of Escherichia coli were obtained from a postmortem tracheal swab. No organisms, including acidfast bacilli and fungi, were demonstrated in impression smears of post-mortem samples of kidney, heart, liver, or brain, and no growth was obtained after aerobic and anaerobic culture for 10 days at $37^{\circ} \mathrm{C}$. and $22^{\circ} \mathrm{C}$. Cultures for Actinomyccs bovis and $M$. tuberculosis also remained sterile. Short, Gram-positive, septate hyphae were demonstrated in impression smears of the lung and spleen. A. fumigatus was cultured from both the lung and a bronchial lymph node. Neither specimen contained acid-alcohol fast bacilli, and $M$. tuberculosis was not isolated.

Lung and lymph node extracts in phosphate-saline buffer, containing penicillin 400 units $/ \mathrm{ml}$., streptomycin $400 \mathrm{mg} . / \mathrm{ml}$., nystatin 100 units $/ \mathrm{ml}$, produced no cytopathogenic effect on monkey kidney and human amnion cultures containing the above antibiotics in onequarter of the above concentrations. Attempts to prepare tissue cultures from lung and lymph node in tissue culture medium were unsuccessful because the preparations were overgrown by $A$. fumigatus in 48 hours.

A small fragment of lung was ground in a Griffith's tube with a few $\mathrm{ml}$. of sterile saline. $0.1 \mathrm{ml}$. and $0.2 \mathrm{ml}$. of the resulting suspension were inoculated intraperitoneally into two guinea-pigs and intravenously into two rabbits. The guinea-pigs remained healthy for 7 weeks. At necropsy their tissues appeared normal and on culture they produced no growth. The rabbits also appeared healthy 4 weeks after inoculation, when that with the larger inoculum was killed. Microscopy and culture of the tissues failed to demonstrate the presence of $A$. fumigatus. The remaining rabbit was challenged intravenously with $1 \mathrm{ml}$. of $A$. fumigatus spores and hyphae in saline (density equalled Brown's scale 3) to determine if it had acquired immunity to infection with this strain of fungus. It died 5 days later of aspergillosis.

Examination of material from the patients' environment. Artificial fertiliser, oilcake, and sweepings from the floor of the barn in which these materials were stored were inoculated directly into a variety of culture media including Sabouraud's glucose agar. $A$ bsidia corymbifera and $A$. fumigatus were isolated from grain seeds in the oilcake. No fungi were recovered from the fertiliser or sweepings.

Characteristics of Aspergillus fumigatus strains. The morphological and cultural properties of the strains isolated from both patients and the oilcake were identical, and typical of $A$. fumigatus. When tested by the disc method using 'Sentest' tablets (Evans Medical Ltd.) all were resistant to penicillin, chlortetracycline, oxytetracycline, chloramphenicol, streptomycin, erythromycin, kanamycin, neomycin, methicillin, and ampicillin, and were sensitive to sulphonamide, nystatin, and amphotericin B.

Rabbit pathogenicity tests. The pathogenicity of the $A$. fumigatus strains from the two patients and the oilcake was tested in three $2.5 \mathrm{~kg}$. rabbits. Each rabbit was inoculated intravenously with $1 \mathrm{ml}$. of a saline suspension of the test culture (density equal to Brown's scale 3). All three rabbits died on the fourth day after inoculation. Multiple minute abscesses were seen throughout the tissues and in each case the renal cortex was particularly affected. Septate hyphae were demonstrated in the lesions and $A$. fumigatus was recovered on culture.

Treatment of experimentally infected rabbits with nystatin and sulphathiazole. Six rabbits each weighing $2.5 \mathrm{~kg}$. were inoculated with $1 \mathrm{ml}$. of the suspension of $A$. fumigatus isolated from Case 2 . The density of the suspension was standardized as above. The first pair of animals, which was untreated, served as controls. The second pair received 50,000 units of nystatin intravenously each day for one week. The nystatin was given as a fine saline suspension prepared from sterile powder supplied by Squibb and Co. for laboratory use only. The third pair of animals received $0 \cdot 1 \mathrm{~g}$. of sulphathiazole (thiazamide sodium, May and Baker) intravenously each day for one week. The two untreated rabbits died of aspergillosis on the fourth day after inoculation. Septate hyphae were demonstrated in the lesions and $A$. fumigatus was recovered on culture. The animals treated with nystatin and sulphathiazole were alive and well 4 weeks after inoculation: they were then killed and necropsy revealed that the tissues of all four animals appeared normal. Microscopsy of impression smears of the liver, spleen, kidney, lungs, and heart in each case failed to reveal the presence of the typical septate hyphae, and $A$. fumigatus was not isolated 
after incubation on Sabouraud's media for 1 week at $37^{\circ} \mathrm{C}$.

\section{Discussion}

Primary aspergillosis of the lungs is difficult to diagnose clinically, and since the fungus is generally regarded as a saprophyte, its presence in postmortem tissues is scarcely incriminating. In the present cases, however, the histological appearances and the demonstration of this fungus, and of no other agent, by histological and bacteriological methods, has led us to believe that these children, who had been in prolonged and close contact with a contaminated environment did, in fact, die of infection with Aspergillus fumigatus.

In the commonest manifestation of pulmonary aspergillosis the fungus forms a localized filamentous network, or aspergilloma, within bronchi, lung cavities, or areas of devitalized lung tissue, without invading the surrounding cellular structures (Hildick-Smith, Blank, and Sarkany, 1964). Apart from small haemoptyses, this usually gives rise to few symptoms, and the fungus is generally regarded as having a saprophytic relationship with the host (Finegold, Will, and Murray, 1959). Alternatively, aspergillus may infect the bronchi, spreading over the bronchial mucosa without penetrating it and may give rise to symptoms associated with hypersensitivity (Hinson, Moon, and Plummer, 1952). Pulmonary invasion by the fungus is the least common form and has usually been reported in the presence of pre-existing lung disease, severe metabolic disturbance, or a generalized disorder, particularly one involving the lympho-reticular system (Symmers, 1964). Treatment with corticosteroids or broad spectrum antibiotics may also predispose to fungal infection (Sidranski and Pearl, 1961). Although only a single example of this secondary form of invasive pulmonary aspergillosis was discovered in a two-year survey of fungus disease in Britain (Duncan, 1945), there is reason to believe that it has since become more common (Symmers, 1964).

Review of published reports. Primary invasive pulmonary infection with any of the species of aspergillus appears to be rare at any age but is especially rare in children. Only a few of the reported cases may be regarded as having been established on the clinical and pathological evidence presented. The first description of a rapidly fatal outcome in children (Hertzog, Smith, and Goblin, 1949) was of a previously healthy boy of 5 years and his sister of 11 years who, like our patients, lived on a farm. Within a very short time of one another, these children developed an acute pneumonic illness with radiological diffuse pulmonary infiltration. Cultures from the throat, blood, and aspirated lung material showed no growth. Penicillin, streptomycin, and sulphadiazine were ineffective, and they died within one month. Necropsy which was performed only on the boy, showed the lungs to be studded with small whitish granulomatous nodules from which $A$. fumigatus was cultured as the sole pathogen. Despite the failure to obtain a necropsy, his sister probably died from the same disease.

A further report concerned an infant, also living on a farm, who died at the age of 20 days from bronchopneumonia and meningitis after an illness lasting only one week. At necropsy, multiple granulomatous lesions were found in the lungs and meninges in which colonies of mycelium and spores were identified as an aspergillus (Akkoyunlu and Yucel, 1957).

A third report described an infant who died of aspergillosis when 18 days old (Allan and Anderson, 1960). Death was preceded by a widespread papular rash, cyanotic attacks, and hepatomegaly, and at necropsy there were multiple, circumscribed granulomatous nodules in the lungs, spleen, and kidneys. Histologically, granulomatous foci were also found in the skin, heart, duodenum, thyroid, tongue, and in a peribronchial lymph node. $A$. fumigatus was identified in these lesions by morphology and culture.

A chronic form of primary aspergillosis has been reported in children. Cawley (1947) described a child of 7 who had recurrent pneumonic episodes from the age of 2 weeks, and whose illness was complicated by an abscess of the thoracic wall and of the cerebellum from both of which $A$. fumigatus was cultured. The lungs at necropsy showed extensive granulomatous involvement, and widespread dissemination to many tissues had occurred as a terminal event. Likewise, Tobler and Minder (1954) described chronic granulomatous pulmonary lesions in a boy of 6 years who died following the surgical removal of an aspergillus granuloma of the cerebellum. Dissemination had also occurred to the bones and lymph nodes.

One juvenile case with recovery, reported by Conen, Walker, Turner, and Field (1962) was in a girl of 7 years whose illness followed the inhalation of timothy grass. She developed an area of pulmonary infiltration which gradually spread to other parts of the lung and was complicated by a chest wall abscess which yielded $A$. fumigatus in pure culture. An apparently metastatic frontal lobe abscess containing mycelia was later removed. A five-week course of intravenous amphotericin $B$ was given one 
year after the start of her illness, but she did not finally recover until three years later.

A primary pulmonary lesion was not found by Iyer, Dodge, and Adams (1952) in their report of a fatal chronic granulomatous meningo-encephalitis in an infant, ascribed to an aspergillus which was identified morphologically in the lesions. Clinically the infant, who had previously been normal, developed mental deterioration, spasticity, and convulsions between the ages of 4 and 11 months.

In the rare cases of primary pulmonary aspergillosis which have been reported in adults, the clinical and pathological picture closely resembles that seen in childhood. Thus Grekin, Cawley, and Zheutlin (1950), Grcevic and Matthews (1959), and Orie, De Vries, and Kikstra (1960) described acute cases, all of which ended fatally, whereas Finegold et al. (1959) and Braatelien and Perlmutter (1961) reported patients with chronic, slowly progressive pulmonary disease.

Clinical picture. From the reported cases and our own experience, it appears that pulmonary aspergillosis in childhood can be a fatal acute pneumonic illness. In infancy, it may be fulminating, with death in a single week, and none of the children with the acute form have survived for longer than 4 weeks. The infection appears to be unaffected by a wide range of antibacterial therapy. Widespread dissemination outside the lungs may occur and may give rise to pustular skin lesions, hepatomegaly, or meningitis; a fungal endocarditis has also been reported in generalized aspergillosis (Luke, Bolande, and Gross, 1963). Although there may be a neutrophil leucocytosis, pyrexia did not exceed $100^{\circ} \mathrm{F} .\left(37 \cdot 8^{\circ} \mathrm{C}\right.$.) in either of our patients, and fever does not seem to have been a striking feature in the other reported cases. The radiological picture appears to be that of a diffuse, fine, or coarse, fluffy-edged mottling of the lung fields. This appearance is not, of course, specific for aspergillosis and may be seen in invasive pulmonary disease due to other fungi, pulmonary tuberculosis, or in other lung diseases.

The cases reviewed indicate that primary pulmonary aspergillosis may also take a chronic course. Localized granulomatous pulmonary lesions occur which may enlarge gradually, or spread to affect other parts of the lungs. The chest wall may be involved, with the formation of an abscess, and isolated metastatic lesions, notably to the brain, can also develop. Widespread dissemination to many tissues may occur as a terminal event.

Pathology. The necropsy finding of widespread pale nodular lesions in the lungs should suggest the possibility of an acute fungal infection, and in the cases reviewed the nodules have been granulomata similar to those we have described. A tendency for the fungus to invade blood vessels was observed histologically by Allan and Anderson (1960) and clearly may lead to metastatic spread.

Aetiology. The histological features and distribution of the lesions in the lungs indicate that inhalation was the probable route of infection, though septicaemic spread from the paronychia, which both children had before their illness, is a remote possibility.

Treatment of the second child with prednisone, and of both children with antibacterial therapy, may have modified their response to the fungus. Nevertheless, as the infection was established clinically and radiologically before these were administered, they clearly played no part in initiating pulmonary invasion.

Diagnosis. Because of its rarity, primary infection with aspergillus may not initially be suspected. If considered, it may be extremely difficult to obtain confirmation by means of positive cultures from the patient during life. In none of the acute cases in childhood, which have been reviewed, was the fungus isolated before necropsy, and this was also our own experience. However, acute invasive pulmonary aspergillosis, in addition to other fungal infections, should be included in the differential diagnosis of a puzzling acute pneumonic illness, particularly if accompanied by radiological evidence of diffuse mottling of the lung fields and a neutrophil leucocytosis. Suspicion should be strengthened if there is a lack of response to all forms of antibacterial therapy. A similar, but usually less acute, clinical illness and radiological picture may be produced by such rare disorders as Pneumocystis carinii pneumonia, pulmonary alveolar proteinosis, acute interstitial pulmonary fibrosis, and acute beryllium pneumonitis. These conditions are also characterized by negative bacteriological tests and lack of therapeutic response.

An adequate lung biopsy may be the only way of establishing the diagnosis, but the risks of performing this in an acutely ill patient may be serious. Although skin testing with aspergillus extracts may be of value in the diagnosis of the hypersensitivity type of broncho-pulmonary aspergillosis (Pepys, Riddell, Citron, Clayton, and Short, 1959), the value of skin testing in the diagnosis of acute invasive aspergillosis has not been established.

When faced with the possibility of this acute 
infection and the prospect of a fatal outcome, the clinician might consider a therapeutic trial of one of the newer antifungal antibiotics, though the treatment of such fungal infections as coccidioimycosis with amphotericin $B$ has shown that the technical problems of drug administration are difficult (see below), and that serious, and even fatal toxic, effects may occur (Ziering and Rockas, 1964).

Treatment. Experience of the treatment of invasive pulmonary aspergillosis with antifungal antibiotics has been limited by the rarity of the disease. Nystatin is essentially for local use and has been of value in infections involving the pleural cavity (Manning and Robertson, 1959). Its use, however, may be extended to pulmonary infection by means of aerosol inhalations (Riddell, 1956), or intrabronchial instillations (Ragaini and Tosi, 1959). The newer polyene antibiotic, natamycin, is similarly confined to topical use and has also been of value as an aerosol in non-invasive forms of bronchopulmonary aspergillosis (Edwards and La Touche, 1964). Amphotericin $B$ is probably the most effective antibiotic for the treatment of systemic aspergillosis, though to achieve a therapeutic effect, intravenous administration, possibly for a number of weeks, may be necessary. The considerable risk of toxic effects may be reduced by a graduated increase in dosage and administration by dilute infusion (Newcomer, Sternberg, Wright, Reisner, McNall, and Sorenson, 1960).

X5079C (Hoffman La Roche) may also be of value in invasive aspergillosis (Utz, Andriole, and Emmons, 1961), and this drug has the advantage of subcutaneous administration.

Hyperbaric oxygen may possibly prove to be a valuable adjunct to other forms of treatment. McAllister, Stark, Norman, and Ross (1963) demonstrated that oxygen at two atmospheres pressure is strongly bacteriostatic to cultures of $A$. fumigatus. A penguin at the London Zoo was recently cured of pulmonary aspergillosis, diagnosed on clinical grounds, after exposure to two atmospheres of oxygen for an hour daily for a week (W. I. Hopkinson and K. G. Williams, 1965, personal communication). As invasive pulmonary aspergillosis in these birds is almost invariably fatal, as it is in man, it is suggested that this form of therapy should be seriously considered in human aspergillosis.

It is clear that invasive pulmonary aspergillosis presents both a diagnostic and a therapeutic challenge. If, as seems likely, the incidence of fungal diseases is increasing, a greater awareness of the possibility of these disorders appears to be desirable.

\section{Summary}

Rapidly fatal pulmonary infection with Aspergillus fumigatus is described in a previously healthy girl of 4 years and her sister of 14 months from a farming family.

Both children were severely dyspnoeic and showed diffuse pulmonary mottling radiologically. Bacteriological tests in life were negative. No response was observed to antibacterial therapy.

Widespread granulomatous inflammation of the lungs was found at necropsy in both patients and $A$. fumigatus was identified in the lesions. An apparently identical strain of this fungus was recovered from cattle-cake kept where the children had played.

Cases of primary invasive pulmonary aspergillosis previously reported are briefly reviewed.

The difficulties of diagnosis and treatment of this rare infection are discussed.

We wish to express our thanks to Professor J. L. Henderson and Professor A. C. Lendrum for their advice and criticism, and to Mr. R. Fawkes, A.I.M.L.T., who undertook the photography.

\section{REFERENCES}

Akkoyunlu, A., and Yucel, F. A. (1957). Aspergillose bronchopulmonaire et encéphalo-méningée chez un nouveau-né de 20 jours. Arch. franc. Pédiat., 14, 615.

Allan, G. W., and Anderson, D. H. (1960). Generalized aspergillosis in an infant 18 days of age. Pediatrics, 26, 432.

Braatelien, N. T., and Perlmutter, H. M. (1961). Aspergillosis of the lung. Dis. Chest, 39, 425.

Cawley, E. P. (1947). Aspergillosis and the aspergilli. Arch. intern. Med., 80, 423.

Conen, P. E., Walker, G. R., Turner, J. A., and Field, P. (1962). Invasive primary aspergillosis of the lung with cerebral metastasis and complete recovery. Dis. Chest, 42, 88.

Duncan, J. T. (1945). A survey of fungous diseases in Great Britain. Results from the first eighteen months. Brit. med. F., $2,715$.

Edwards, G., and La Touche, C. J. P. (1964). The treatment of bronchopulmonary mycoses with a new antibiotic - pimaricin. Lancet, 1, 1349.

Finegold, S. M., Will, D., and Murray, J. F. (1959). Aspergillosis. A review and report of 12 cases. Amer. F. Med., 27, 463.

Grcevic, N., and Matthews, W. F. (1959). Pathologic changes in acute disseminated aspergillosis. Amer. F. clin. Path., 32, 536.

Grekin, R. H., Cawley, E. P., and Zheutlin, B. (1950). Generalized aspergillosis: report of a case. Arch. Path., 49, 387.

Grocott, R. G. (1955). A stain for fungi in tissue sections and smears using Gomori's methenamine-silver nitrate technic. Amer. F. clin. Path., 25, 975.

Hertzog, A. J., Smith, T. S., and Goblin, M. (1949). Acute pulmonary aspergillosis. Pediatrics, 4, 331.

Hildick-Smith, G., Blank, H., and Sarkany, I. (1964). Fungus Diseases and their Treatment, p. 336. Churchill, London.

Hinson, K. F. W., Moon, A. J., and Plummer, N. S. (1952). Broncho-pulmonary aspergillosis: $A$ review and a report of eight new cases. Thorax, 7, 317 .

Iyer, S., Dodge, P. R., and Adams, R. D. (1952). Two cases of aspergillus infection of the central nervous system. $\mathcal{F}$. Neurol. Neurosurg. Psychiat., 15, 152. 
Luke, J. L., Bolande, R. P., and Gross, S. (1963). Generalized aspergillosis and aspergillus endocarditis in infancy. Pediatrics, 31, 115.

Manning, L. K., and Robertson, L. (1959). A case of aspergillosis treated with nystatin. Brit. med. $\mathcal{f}_{\text {., }} 1,345$.

McAllister, T. A., Stark, J. M., Norman, J. N., and Ross, R. M. (1963). Inhibitory effects of hyperbaric oxygen on bacteria and fungi. Lancet, 2, 1040.

Newcomer, V. D., Sternberg, T. H., Wright, E. T., Reisner, R. M., McNall, E. G., and Sorenson, L. J. (1960). The treatment of systemic fungus infections with amphotericin B. Ann. N.Y. Acad. Sci., 89, 221.

Orie, N. G. M., De Vries, G. A., and Kikstra, A. (1960). Growth of aspergillus in the human lung. Aspergilloma and aspergillosis. Amer. Rev. resp. Dis., 82, 649.

Pepys, J., Riddell, R. W., Citron, K. M., Clayton, Y. M., and Short, E. I. (1959). Clinical and immunologic significance of aspergillus fumigatus in the sputum. ibid., 80, 167.

Ragaini, S., and Tosi, C. (1959). Su di un caso di micetoma polmonare guarito mediante broncoinstillazioni antimicotiche. Minerva med., 50, 105.
Riddell, R. W. (1956). Fungous diseases of Britain. Brit. med. F., 2, 783.

Sidranski, H., and Pearl, M. A. (1961). Pulmonary fungus infections associated with steroid and antibiotic therapy. Dis. Chest., 39, 630.

Symmers, W. St. C. (1964). The occurrence of deep-seated fungal infections in general hospital practice in Britain today. Proc. roy. Soc. Med., 57, 405.

Thom, C., and Raper, K. B. (1945). A Manual of the Aspergilli, p. 150. Williams and Wilkins, Baltimore.

Tobler, W., and Minder, W. (1954). Generalisierte chronische aspergillose beim kind und ihre Beziehung zur antibiotischen therapie. Helv. paediat. Acta, 9, 209.

Utz, J. P., Andriole, V. T., and Emmons, C. W. (1961). Chemotherapeutic activity of $\mathrm{X}-5079 \mathrm{C}$ in systemic mycoses of man. Amer. Rev. resp. Dis., 84, 514.

Ziering, W. H., and Rockas, H. R. (1964). Coccidioidomycosis: long-term treatment with amphotericin $\mathbf{B}$ of disseminated diseases in a three-month-old baby. Amer. F. Dis. Child., 108, 454. 